\title{
Foreword to the Special Focus on Automated Deduction in Geometry
}

\author{
Xiaoyu Chen · Hongbo Li · Jing Yang
}

Published online: 25 June 2020

(C) Springer Nature Switzerland AG 2020

Getting theorems proved on machine has been dreams of mathematicians and computer scientists for centuries. For geometry, highly successful methods for theorem proving and discovering have been proposed as research advances from the development of Gelernter's theorem proving machine to computer-aided proof of the four-color theorem and to mechanized proving of hundreds of theorems using Wu's method. The success of machine-supported theorem proving in geometry is kept continuing, for example, with formally verified proof of the Four Color theorem, complete formal proof of the Kepler conjecture and automated generation of hundreds of theorems from images of diagrams. Along with these remarkable achievements, sophisticated mathematical theories, reasoning methodologies, computer algorithms and software tools have been developed by many prominent scientists, which not only make machines more and more powerful and intelligent for geometry theorem proving and discovering but also contributes significantly to the advances of many relevant research subjects.

The series of international workshops on Automated Deduction in Geometry (ADG) is a forum dedicated to the exchange of ideas and views, to the presentation of research results and progress, to the discussion of theoretical and practical challenges and to the demonstration of software tools on the intersection between geometry, computation and automated reasoning. The twelfth in this series of biennial international workshops on ADG was held in Nanning, China, during September 11-14, 2018, in memory of Wen-tsün Wu, founder of the Chinese school of mathematics mechanization.

After ADG 2018, we launched an open call for papers inviting workshop participants and other involved people in the ADG community to participate in a special focus of Mathematics in Computer Science. After a detailed peer-review process according to the standard refereeing procedure of the journal, we selected an invited survey paper and four full research papers, which show the trend set and applicability of current research in automated reasoning in geometry. We complete the foreword by a brief introduction to these papers.

\section{Chen $(\varangle)$}

SKLSDE-BDBC-LMIB, School of Mathematical Sciences, Beihang University, Beijing 100191, China

e-mail: chenxiaoyu@buaa.edu.cn

H. Li

Key Lab of Mathematics Mechanization, Academy of Mathematics and Systems Sciences, Chinese Academy of Sciences, Beijing 100080, China

e-mail: hli@mmrc.iss.ac.cn

J. Yang

SMS, School of Mathematics and Physics, Guangxi University for Nationalities, Nanning 530006, China

e-mail: yangjing0930@gmail.com 
The invited survey paper by Pedro Quaresma firstly introduces the methods, techniques and software systems that have been studied and developed for automatically proving and discovering geometric theorems in the past 5 decades and then addresses research advances of applying automated deduction tools for geometric knowledge accumulation and dissemination, including formal proof readability, efficiency comparison and information exchange of different geometric theorem provers, repositories of geometric knowledge with powerful search mechanisms and knowledge classification and management. Finally, a perspective of an adaptive, correct, collaborative, visual and intelligent geometry book is described to indicate research and development challenges in the future.

Philip Todd proposes a new method, called Analytical Geometry Method, for automated theorem proving in geometry, discusses how it has been integrated into a (author's) tool Geometry Expressions and presents a series of theorems analyzed with the help of this tool. This new prover made it possible for students to explore various relationships between geometric and algebraic models. Despite of strengths and weakness compared with other semi-algebraic automated theorem proving methods, extensive case studies are provided to show its generality.

Zoltán Kovács explores the problem of discovering metric properties on $n$-regular polygons for some integer $n$. The applied theory includes a formula by Watkins and Zeitlin on minimal polynomials of $\cos \frac{2 \pi}{n}$ and a method by Recio and Vélez to discover a property in a plane geometry construction. The method is based on Wu's idea of algebraizing geometric constructions and utilizes the theory of Gröbner bases for precisely symbolic computation. A numerical check method is also implemented with GeoGebra for fast exploration of possible properties.

Dongming Wang, Bo Huang and Xiaoyu Chen approach an interesting question of whether there exists any equilateral triangle, other than Morley triangles, which is formed by three intersection points of the neighbouring angular $n$-sectors of an arbitrary triangle $\Delta$ for $n>3$. Specialized techniques with interactive, semi-automatic algebraic computations are used to prove that for $n=4$ and 5 the three points, each of which is the intersection of the two internal (or two external) angular $n$-sectors closest to the same side of $\Delta$, form an equilateral triangle if and only if $\Delta$ is equilateral. The proposed computational approach can be also applied to other cases for specific $n$.

Philip Todd also presents a system, called Mechanical Expressions, for automated formula discovery in engineering mechanics built on Lagrangian formulation where geometric constraints are treated as physical constraints. A software architecture is described for the system which layers Lagrangian mechanics on top of a geometric constraint model to generate formulas in engineering mechanics. Various user interfaces are designed for different mechanical settings. A list of examples is also provided from kinematics, statics and dynamics of simple mechanisms to illustrate different mechanical features and show the capacity of the system.

Finally, we would like to thank the reviewers, both from the Program Committee of ADG and external referees, for their efforts in the process of selection and improvement of the submitted papers. We would also like to acknowledge the help from MCS editorial team.

Publisher's Note Springer Nature remains neutral with regard to jurisdictional claims in published maps and institutional affiliations. 